\title{
Permeability as an important parameter for radon risk classification of foundation soils
}

\author{
Matej Neznal and Martin Neznal \\ Radon v.o.s. Corp., Prague, Czech Republic
}

\begin{abstract}
Permeability of soils and rocks for gases is one of the main parameters for final radon risk classification of building sites. For the improvement of the method for radon risk classification it is recommended to complete in situ measurements of permeability and/or results derived from grain size analysis by an expert description of parameters of soils and rocks and their changes in vertical profile.
\end{abstract}

Key words permeability - radon - soil gas - transfer factor

\section{Introduction}

The uniform method, that has been used for radon risk classification in the Czech Republic since 1994 (Barnet, 1994), is based on the assessment of the soil-gas radon $\left({ }^{222} \mathrm{Rn}\right)$ concentration and of the permeability of soil and rock for gases. Both parameters are evaluated in the vertical profile down to the level of assumed building foundations or to the level of assumed contact building-soil. Results of detailed radon surveys as well as results obtained from research studies carried out in the Czech Republic in the period 1994-2000 indicated that the uniform method should be improved. There are many problems connected with the determination of soil permeability itself and some correspond to a variable understanding of what this parameter describes or what it means in fact.

Mailing address: Dr. Matej Neznal, Radon v.o.s. Corp., Novakovych 6, 180 00, Prague 8, Czech Republic; e-mail: neznal@clnet.cz
Due to the original method for classification it is possible to use direct in situ measurements of permeability based on soil gas withdrawal by means of low negative pressure (Kašpar et al., 1993; Barnet, 1994; Neznal et al., 1995) or particle size analyses (the permeability is derived very simply from the weight percentage of fine fraction in the soil sample, Barnet, 1992; Neznal et al., 1992).

These data are completed with the description of changes in vertical profiles with respect to the expected foundation depth of the building. The main disadvantages are given by the fact that in case of particle size analyses other factors influencing the permeability (natural soil moisture, density, effective porosity, etc) are not taken into consideration.

Furthermore, one sample cannot describe a heterogeneous geological environment (horizontal and vertical changes, influence of human activity). In case of direct measurements the results are strongly dependent on small scale variations of the character of the soil, and the equipment commonly used in the Czech Republic does not enable us to measure exactly in extremely low and/or high permeable soils (results vary in several orders of magnitude). 


\section{Methods and results}

At first a wide range of methods for soil-gas permeability determination in radon research was considered for further investigation - mainly single probe measurements (Damkjaer and Korsbech, 1992), dual probe measurements (Garbesi et al., 1993) and derivation of permeability from other parameters - permeability for water (Rogers and Nielson, 1991), grain size analysis (Barnet, 1992; Neznal et al., 1992), etc. After comparison of advantages and disadvantages of different systems with respect not only to professional issues, but also to economic ones, three prototypes for direct measurements were prepared. The main goal was to avoid or decrease the disadvantages of RADONJOK equipment (single probe system that is used in Czech Republic, Kašpar et al., 1993; Neznal et al., 1995).

RADON-JOK is a portable piece of equipment which has been developed for in situ measurements of gas permeability of soils. Its robustness and simplicity make it very practical for easy, quick and at the same time sufficiently exact in situ investigation. The principle of the RADON-JOK equipment is based on air withdrawal by means of negative pressure. The air is pumped from the soil under constant pressure through a specially designed probe with a constant surface of contact between the probe head and the soil. The constant active area is created in the head of the probe (driven into the soil to a measured depth) by the extrusion of the tip by means of the punch wire inside the probe by an exact distance. The special rubber sack, with one or two weights, pumps the air from the soil and allows measurements to be made at very low pressures. The permeability is calculated from the equation using the known air flow through the probe. The air flow is defined by the known air volume $(=2000 \mathrm{ccm})$ in the rubber sack (depression of the bottom of the sack between two notches) and by the pumping time measured. The great advantage of RADON-JOK is the possibility to perform measurements independently of any source of energy (electricity, compressed air). Using the RADON-JOK system, with the shape factor $F=0.149 \mathrm{~m}$, the feasible range of measured permeabilities is approxi- mately from $10^{-11}$ to $10^{-14} \mathrm{~m}^{2}$. The maximum detection limit (measured time about $8 \mathrm{~s}$ ) is determined by the resistance of the equipment, the minimum detection limit depends on the time spent by any given measurement (usually used near $1.10^{-14} \mathrm{~m}^{2}$ ).

Three prototypes and RADON-JOK system were tested at several test sites with different geological conditions. The tests confirmed the expected limitations of particular approaches. The range of real gas permeabilities is so large, that it is almost impossible to find a way to measure precisely with the same equipment in a low permeable environment (in case of pressure systems it means higher pressure differences, longer time and lower air flow) and in a high permeable one (just opposite situation). Single probe system RADON-JOK was chosen for testing of temporal and spatial variability due to its simplicity and possibility to compare with previous results.

The measurements were performed at four reference areas with respect to various geological conditions each month during a one-year period. Permeability of soils and rocks for gasses was measured at 15 points at each reference area. At two reference areas (Lysa nad Labem, Kocanda) the measurements were realized at each point at two depths, 0.4 and $0.8 \mathrm{~m}$, and at two reference areas (Svetice, Klanovice) at a depth of $0.8 \mathrm{~m}$ only. The soil gas samples for soil gas radon concentration measurements (Neznal et al., 1996) were collected at the same depths and to the same extent. Other important parameters, mainly soil

Table I. Correlation coefficients at the area Lysa nad Labem.

\begin{tabular}{cc}
\hline \hline $\begin{array}{c}\text { Correlation between } \\
\text { parameter }(\text { depth })\end{array}$ & $\begin{array}{c}\text { Correlation } \\
\text { coefficient }\end{array}$ \\
\hline$c_{\mathrm{Rn}}(0.8 \mathrm{~m}) / c_{\mathrm{Rn}}(0.4 \mathrm{~m})$ & 0.92 \\
$k(0.8 \mathrm{~m}) / k(0.4 \mathrm{~m})$ & 0.83 \\
$c_{\mathrm{Rn}}(0.8 \mathrm{~m}) / k(0.8 \mathrm{~m})$ & -0.68 \\
$c_{\mathrm{Rn}}(0.4 \mathrm{~m}) / k(0.4 \mathrm{~m})$ & -0.60 \\
$c_{\mathrm{Rn}}(0.8 \mathrm{~m}) / w(0.1 \mathrm{~m})$ & 0.70 \\
$c_{\mathrm{Rn}}(0.8 \mathrm{~m}) / w(0.25 \mathrm{~m})$ & 0.88 \\
$k(0.8 \mathrm{~m}) / w(0.1 \mathrm{~m})$ & -0.52 \\
$k(0.8 \mathrm{~m}) / w(0.25 \mathrm{~m})$ & -0.66 \\
\hline
\end{tabular}


moisture (Kuráž and Matoušek, 1997) and/or the mass percentage of fractions, porosity and water saturation were determined with respect to Czech National Standards.

In general, good correlations between the measured parameters were obtained in the areas with homogeneous and high permeable soils. These relations can be illustrated by the correlation coefficients between median values of gas permeability $(k)$, soil gas radon concentration $\left(c_{\mathrm{Rn}}\right)$ and soil moisture $(w)$ at the reference area Lysa nad Labem (table I). As for the geological conditions, the bedrock is formed by Cretaceous sediments and the Quarternary cover by fluvial sands at the area. The upper soil layers are homogeneous, characterized by low

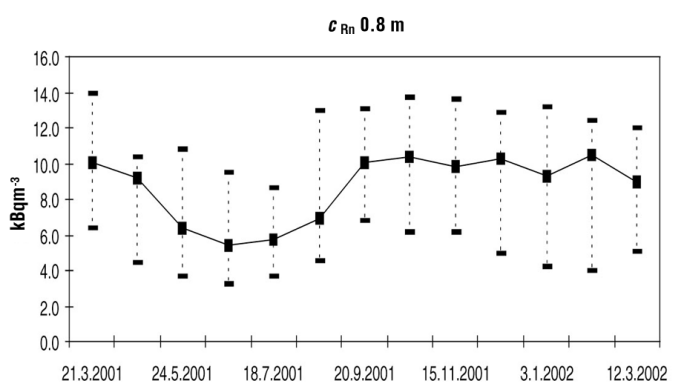

(a)

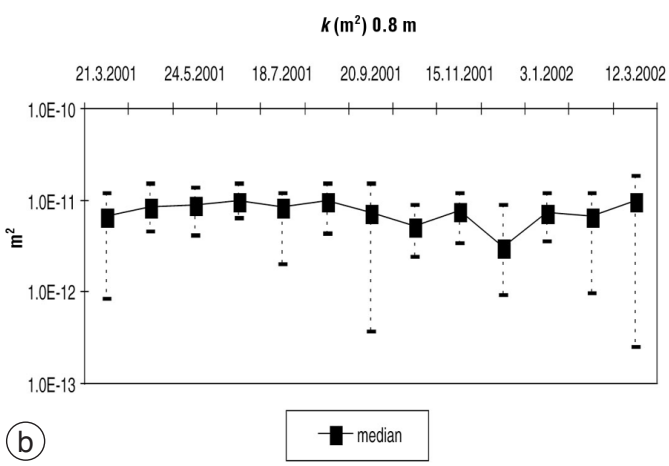

Fig. 1a,b. Temporal and spatial variations of soil gas radon concentration $c_{\mathrm{Rn}}$ and permeability $k$ in the depth of $0.8 \mathrm{~m}$ at the area Lysa nad Labem - minimal, median and maximal values of 15 measurements. values of soil gas radon concentration and high permeability (fig. 1a,b).

Values of the correlation coefficient indicate a positive correlation between the soil gas radon concentration at a depth of $0.8 \mathrm{~m}$ and the soil moisture in surface horizons and a negative correlation between the soil gas radon concentration and permeability and between the permeability and soil moisture. Positive correlation was confirmed between the soil gas radon concentration determined at two different depths. The same conclusion was valid for the permeability values measured at two different depths.

On the other hand a correlation between the soil-gas radon concentration and the permeability, as well as between the permeability, soil moisture and other parameters can be often very weak at areas with medium or low permeable environment. It can be illustrated by the results obtained at the reference area Kocanda (see fig. $2 \mathrm{a}, \mathrm{b})$. This area lies in the region of Proterozoic paragneiss covered by Quarternary loess and loess loams. Although it can be described as an area with homogeneous upper soil layers, observed spatial and vertical changes in permeability do not allow us to confirm any relation between measured parameters. The single values of direct in situ measurements of permeability as well as soil moisture are substantially affected by the small scale changes in conditions in measured soil volume, especially in case of medium and low permeable environment. Although the layers seem to be homogeneous, the in situ measurements characterizing small soil elements result in a large spatial variability. The statistical evaluation of permeability measurements is affected by an occurrence of high permeable outsiders on one hand and of values under the minimum detection limit in case of extremely low permeability on the other. A wide variability of results obtained on small scale conditions makes any evaluation very difficult.

A similar conclusion was valid even for the high permeable environment, when relatively high water saturation of upper horizons occurred (reference area Klanovice). The changing water content caused large spatial variability of permeability results too.

The spatial variability of permeability, expressed as a ratio $\mathrm{SD} /$ mean, ranged typically 


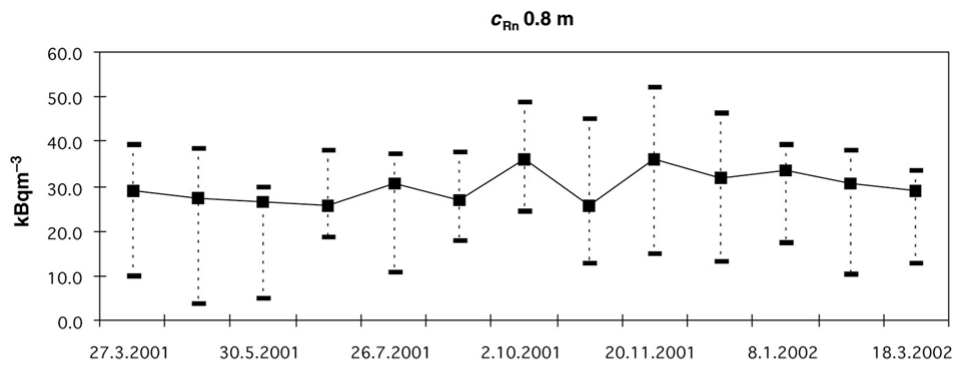

(a)
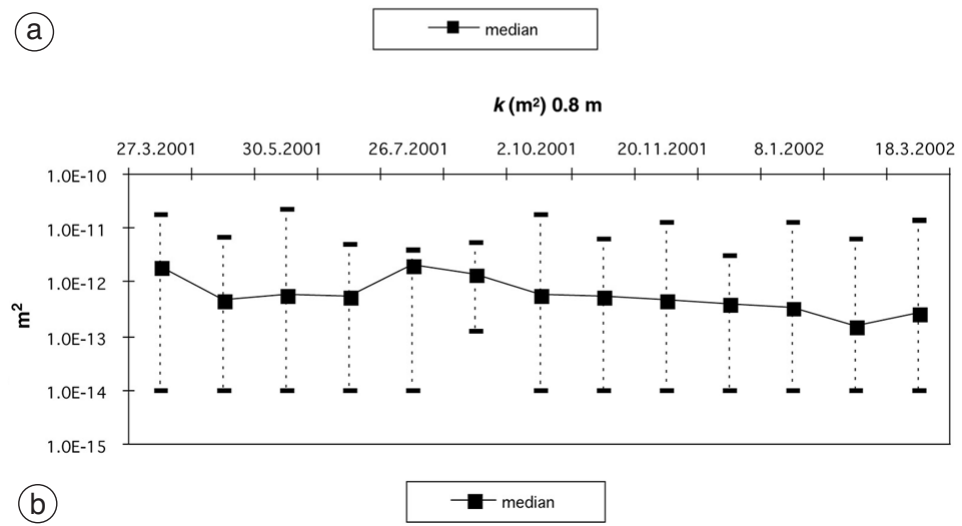

Fig. 2a,b. Temporal and spatial variations of soil gas radon concentration $c_{\mathrm{Rn}}$ and permeability $k$ in the depth of $0.8 \mathrm{~m}$ at the area Kocanda - minimal, median and maximal values of 15 measurements.

from 50 to $200 \%$ at the area Kocanda, Klanovice, Svetice and from 25 to $50 \%$ at the area Lysa nad Labem with high permeable homogeneous environment, respectively.

These follow-ups were completed by measurements of permeability and of soil-gas radon concentration in various vertical profiles with substantial changes in permeability to be able to describe the influence of permeability changes on radon concentration with depth and to classify possible errors connected with the application of the uniform depth for permeability measurements.

Three areas were chosen for these measurements. Two of them (Kostelec, Sedlcanky) with the increasing permeability in the vertical profile with depth (low permeable upper horizon the depth $0.4 \mathrm{~m}$, medium permeable horizon at the depth $0.8 \mathrm{~m}$ and high permeable layer at the depth of $1.1 \mathrm{~m}$ ). The last area Skochovice had the low permeability along a vertical profile with some small changes - a slow decrease with depth. Soil-gas radon concentration measurements and in situ permeability measurements were performed at 15 points and at three depths $(0.4 \mathrm{~m}, 0.8 \mathrm{~m}$ and $1.1 \mathrm{~m})$ in each area.

Almost no changes in soil gas radon concentration with depth were observed. This can be illustrated by the variability of soil gas radon concentration $\left(c_{\mathrm{Rn}}\right)$ and permeability $(k)$ with depth (range of measured values in the specified depth and median value) at the reference areas (table II).

The increase in radon concentration with depth could be presumed at two areas with substantial changes in permeability in the vertical profile (Sedlcanky, Kostelec). But this expectation was not confirmed, dramatic changes as well as local anomalies of radon concentration caused by a sealing effect of upper layers with 
Table II. Variability of soil gas radon concentration $c_{\mathrm{Rn}}$ and permeability $k$ with depth.

\begin{tabular}{|c|c|c|c|c|}
\hline Depth (m) & $c_{\mathrm{Rn}}\left(\mathrm{kBqm}^{-3}\right)$ & Median $c_{\mathrm{Rn}}\left(\mathrm{kBqm}^{-3}\right)$ & $k\left(\mathrm{~m}^{2}\right)$ & Median $k\left(\mathrm{~m}^{2}\right)$ \\
\hline \multicolumn{5}{|l|}{ Sedlcanky area } \\
\hline 0.4 & $11.7-38.0$ & 21.4 & $9.9 \cdot 10^{-15}-7.3 \cdot 10^{-13}$ & $9.9 \cdot 10^{-15}$ \\
\hline 0.8 & $14.9-36.4$ & 21.9 & $7.6 \cdot 10^{-14}-1.1 \cdot 10^{-11}$ & $6.5 \cdot 10^{-13}$ \\
\hline 1.1 & $14.0-27.6$ & 18.8 & $1.0 \cdot 10^{-13}-1.6 \cdot 10^{-11}$ & $7.2 \cdot 10^{-12}$ \\
\hline \multicolumn{5}{|l|}{ Kostelec area } \\
\hline 0.4 & $24.7-38.7$ & 32.7 & $9.9 \cdot 10^{-15}-1.7 \cdot 10^{-12}$ & $1.2 \cdot 10^{-13}$ \\
\hline 0.8 & $26.3-39.9$ & 32.7 & $9.9 \cdot 10^{-15}-1.1 \cdot 10^{-11}$ & $5.8 \cdot 10^{-13}$ \\
\hline 1.1 & $29.2-43.3$ & 32.8 & $7.2 \cdot 10^{-13}-1.1 \cdot 10^{-11}$ & $7.0 \cdot 10^{-12}$ \\
\hline \multicolumn{5}{|l|}{ Skochovice area } \\
\hline 0.4 & $39.3-100$ & 66.1 & $9.9 \cdot 10^{-15}-3.9 \cdot 10^{-12}$ & $1.8 \cdot 10^{-13}$ \\
\hline 0.8 & $44.4-104$ & 74.6 & $9.9 \cdot 10^{-15}-1.1 \cdot 10^{-11}$ & $8.3 \cdot 10^{-14}$ \\
\hline 1.1 & $29.8-101$ & 74.3 & $9.9 \cdot 10^{-15}-9.1 \cdot 10^{-12}$ & $9.9 \cdot 10^{-15}$ \\
\hline
\end{tabular}

lower permeability did not appear. At the area Skochovice, similar values of radon concentration were determined in the depths of $0.4 \mathrm{~m}$ and $1.1 \mathrm{~m}$, although the upper layers were more permeable and lower values could be therefore expected. The influence of permeability changes on changes of radon concentration with depth seems to be low (Neznal et al., 1996).

\section{Conclusions and recommendations}

The classification of permeability based only on direct in situ measurements of permeability or on particle size analyses can be misleading. As one sample or one result cannot often describe a heterogeneous geological environment (horizontal and vertical changes, influence of human activity, small scale variations in the character of the soil), it would be necessary to increase substantially the number of measurements (and the costs of classification). But large spatial variability of results could make any statistical evaluation rather difficult and in some cases it could cause the underestimation or overestimation of permeability (changes in soil moisture, significant occurrence macroand micro-fissures, etc.).
The expert responsible for the classification should focus not only on those results of laboratory or in situ tests, but also on the following factors influencing the permeability and their changes in vertical profile from surface down to the level of assumed building foundations or to the level of assumed contact building-soil: grain size distribution, soil moisture, water saturation, effective porosity, porosity, density, bulk density, compactness, thickness of Quarternary cover, weathering character of the bedrock, modification of soil layers by various antropogeneous activities.

To be able to describe the vertical profile, hand drills are usually performed to the desired depth (or to the lowest depth that can be reached by a hand drill, in case of the large amount of coarse fraction or in case of unweathered rock outcrops in the upper layers near the surface). This description, which strongly depends on the expert's personal experience, i.e. on subjective knowledge, should be completed either by a certain number of in situ permeability measurements, or by a subjective evaluation of a resistance during the soil gas sampling for the soil gas radon concentration measurements in all sampling points and by a grain size analysis of representative soil samples.

The expert evaluation of permeability based on the subjective expert assessment of the de- 
scription of changes of those parameters in vertical profile, with the supporting character of results of laboratory or in situ tests, should improve the classification.

Some problems are connected with the fact that the permeability measured (or derived) during the investigation in an undisturbed environment is just the parameter that can be used for determination of radon potential ( risk» point of view). But this permeability would not be the same as the permeability of the environment in the future at the contact between the building and the soil. Therefore it cannot be used without correction for the description of the transfer of radon from soils into the buildings-transfer factor, etc. («transfer» point of view).

\section{Acknowledgements}

The survey was supported by the State Office for Nuclear Safety, Prague.

\section{REFERENCES}

BARNET, I. (1992): Permeability and ${ }^{222} \mathrm{Rn}$ concentration in soil gas - Case studies, in Radon investigation in CS, edited by I. BARnet, Czech Geol. Surv. Prague, 3, 20-23.

BARNET, I. (1994): Radon risk classification for building purposes in the Czech Republic, in Radon investiga- tions in $C R$, edited by I. BARNET and M. NEZNAL, Czech Geol. Surv. Prague, 5, 18-24.

DAmKJAer, A. and U. Korsbech (1992): A small-diameter probe for in-situ measurements of gas permeability of soils, Rad. Prot. Dos., 45 (1/4), 85-89.

Garbesi, K., R.G. SeXtro, W.J. Fisk, M.P. Modera and K.L. REVZAN (1993): Soil-gas entry into an experimental basement: model measurement comparisons and seasonal effects, Environ. Sci. Tec., 27 (3), 466-473.

KaŠPar, J., P. Prokop and M. Matolín (1993): FH-1, equipment for in situ permeability measurements, in Radon investigation in CS, edited by I. BARNET, Czech Geol. Surv. Prague, 4, 4-8.

KuRÁž, V. and J. MATOUŠEK (1997): Water regime of reclamated dumps - Monitoring of soil moisture using dielectric method, in Proceedings of the International Symposium on Soil Behaviour in Time and Space, Wien 1997, 67-70.

Matolín, M. and P. Prokop (1991): Statistical significance of radon determination in soil air, in Radon investigation in CS, edited by I. Barnet, Czech Geol. Surv. Prague, 2, 20-24.

NeZnal, M., M. NeZnAL and J. ŠMARda (1992): Correlation between radon in soil gas and local geology - Some remarks, in Radon investigation in $C S$, edited by I. BARNET, Czech Geol. Surv. Prague, 3, 24-28.

Neznal, M., M. NeznAl and J. SMARdA (1995): Radon risk classification of foundation soils and other radon measurements by private firms, Bulletin Scientifique l'Association Ingénieurs Électriciens Sortis de l'Institut Montefiore, 3, 31-34.

Neznal, M., M. Neznal and J. ŠMarda (1996): Assessment of radon potential of soils - A five-year experience, Environ. Int., 22 (suppl. to no. 1), 819-828.

Rogers, V.C. and K.K. NiELSON (1991): Correlations for predicting air permeabilities and ${ }^{222} \mathrm{Rn}$ diffusion coefficients of soils, Health Phys., 61 (2), 225-230. 